\title{
On (non-) monotonicity of cooperative solutions*
}

\author{
Yair Tauman ${ }^{\dagger}$ \\ Tel Aviv University and Stony Brook University \\ Andriy Zapechelnyuk $k^{\ddagger}$ \\ University of Bonn and KSE-KEI
}

October 22, 2009

\begin{abstract}
Aggregate monotonicity of cooperative solutions is widely accepted as a desirable property, and examples where certain solution concepts (such as the nucleolus) violate this property are scarce and have no economic interpretation. We provide an example of a simple fourplayer game that points out at a class of economic contexts where aggregate monotonicity is not appealing.
\end{abstract}

Keywords: Cooperative games, aggregate monotonicity, axiomatic solution, core, Shapley value, nucleolus

JEL classification: $\mathrm{C} 71, \mathrm{C} 78$

\footnotetext{
*We thank two anonymous referees for helpful comments.

${ }^{\dagger}$ Recanati School of Business, Tel Aviv University, Ramat Aviv, 69978 Tel Aviv, Israel

$\ddagger$ University of Bonn, Economic Theory II, Lennéstrasse 37, 53113 Bonn, Germany. E-mail: zapechelnyuk@hcm.uni-bonn.de
} 


\section{Introduction}

A solution of a cooperative game is said to be aggregate monotonic (Megiddo, 1974) if no player is worse off whenever the worth of the grand coalition increases while the worth of every other coalition remains unchanged. Aggregate monotonicity is a very mild requirement that is broadly considered a desirable and natural property. Among well known solution concepts, the Shapley value, the egalitarian (equal division) rule, ${ }^{1}$ the core (on the class of games with nonempty core) and the per-capita nucleolus (Grotte, 1970; Young et al., 1982) are aggregate monotonic (though the last two violate a slightly stronger requirement of coalitional monotonicity ${ }^{2}$ ), while the nucleolus (Schmeidler, 1969) and the kernel (Davis and Maschler, 1965) are not (see Megiddo, 1974; Hokari, 2000). ${ }^{3}$ Maschler (1992) comments a lack of aggregate monotonicity of the nucleolus:

"This is certainly an undesirable feature, and it bothered some people. One has a feeling that in any "fair" outcome all players should benefit if $v(N)$ [the worth of the grand coalition] increases and other coalitions stay put. For that reason, there was a suggestion (Young et al., 1982) to use the per-capita nucleolus, which yields a monotonic one-point outcome in the core for games with a nonempty core. This is not going to be of much help, because even the per-capita nucleolus does not satisfy a slightly stronger, but not less intuitive coalitional monotonicity property. [...]

\footnotetext{
1 The egalitarian solution equally divides the worth of the grand coalition among the players.

2 A solution is said to be coalitional monotonic (Young, 1985) if for any coalition no member of that coalition is worse off whenever its worth increases while the worth of every other coalition remains unchanged.

${ }^{3}$ Hokari (2000) shows that on the class of convex games the nucleolus is not aggregate monotonic. The same statement applies to the kernel, since on this class of convex games these solution concepts coincide (Maschler et al., 1971).
} 
Surprisingly, Young (1985) proves that for the class of games with nonempty core there does not exist a one-point coalitional monotonic solution which always lies in the core. [...] There is no escape from this fact: if you want a unique outcome in the core, you must face some undesirable monotonicity consequences. On the other hand, if you feel that monotonicity is essential, say, because it "provides incentives" if imposed on a society (Young, 1985), then you should sometimes discard the core, and the nucleolus is not a solution concept that you should recommend." (Maschler, 1992, pp. 613-614)

Whether there is a trade-off between monotonicity and other desirable properties of a solution or not depends on the context from which a cooperative game arises. Some contexts may narrow down the class of games to a subclass where the nucleolus is monotonic. Other contexts may prove the monotonicity requirement completely unreasonable, for instance, Moulin and Thomson (1988) show that in an exchange economy the resource monotonicity of a solution is incompatible with Pareto optimality and some weak requirements of fair division. Aumann (2000) argues:

"Most axioms appearing in axiomatizations do seem reasonable on the face of it, and many of them are in fact quite compelling. The fact that a relatively small selection of such axioms is often categoric (determines a unique solution concept), and that different such selections yield different answers, implies that all together, these reasonable sounding axioms are contradictory. [... Any given kind of a counterintuitive example can be eliminated by an appropriate choice of solution concept, but only at the cost of another quirk turning up. Different solution concepts can therefore be thought of as results of choosing not only 
which properties one likes, but also which examples one wishes to avoid." (Aumann, 2000, p. 77)

Unfortunately, the examples of non-monotonicity of solution concepts in the literature (Megiddo, 1974; Young, 1985; Hokari, 2000) can hardly be put in any economic context. They serve as warnings rather than tools that help one to understand whether the monotonicity property is crucial for one's needs. In this note we present a simple example with a clear economic interpretation where the aggregate monotonicity property of a solution concept is not convincing. The purpose of this note is not just to provide another example where the nucleolus is not aggregate monotonic, or to argue that sometimes the nucleolus is more appealing than the Shapley value. Instead, our main goal is to point out at a certain class of simple economic contexts or settings where the monotonicity property of a solution concept is less appealing than it may seem at first glance.

\section{The Example}

A game in coalitional form (a cooperative game) is a pair $(N, v)$, where function $v$ associates with every coalition of players $S \subset N$ its worth $v(S)$, with the convention $v(\varnothing)=0$. Let $\mathcal{V}$ be a class of games in coalitional form. A solution on class $\mathcal{V}$ is a mapping $\phi$ that maps every game $(N, v) \in \mathcal{V}$ to a payoff vector $\phi(N, v)$ for all players in $N$.

A solution $\phi$ is said to be aggregate monotonic if whenever two games $\left(N, v^{\prime}\right)$ and $\left(N, v^{\prime \prime}\right)$ satisfy $v^{\prime \prime}(N) \geq v^{\prime}(N)$ and $v^{\prime \prime}(S)=v^{\prime}(S)$ for all $S \varsubsetneqq N$, the solution assigns to every player in $v^{\prime \prime}$ at least as high payoff as in $v^{\prime}$, i.e., $\phi_{i}\left(N, v^{\prime \prime}\right) \geq \phi_{i}\left(N, v^{\prime}\right)$ for all $i \in N$.

Let $N=\{0,1,2,3\}$ be the set of players, where player 0 is an employer who possesses a production technology and the other players are employees who use this technology to produce output. The employer on his own can 
produce zero units of output, but if he hires $k$ workers $(k=1,2,3)$, they can produce $f(k)$ units. We define a cooperative game, $(v, N)$, as follows: every coalition that contains either the employer alone or the workers without the employer has zero worth; every coalition that contains the employer and $k$ workers has worth $f(k)$, i.e., $v(S)=f(k)$ if $S \ni 0$ and $|S|=k+1, k=1,2,3$, and otherwise $v(S)=0$.

Let us compare two production functions, $f^{\prime}$ and $f^{\prime \prime}$. The first production function is $f^{\prime}(1)=1$ and $f^{\prime}(2)=f^{\prime}(3)=2$, that is, the total production is the same whether there are two or three workers. This defines a game $\left(N, v^{\prime}\right)$ as follows: $v^{\prime}(0, i)=1$ and $v^{\prime}(0, i, j)=v^{\prime}(N)=2$ for all distinct $i, j \in\{1,2,3\}$, and $v^{\prime}(S)=0$ otherwise. The second production function is $f^{\prime \prime}(k)=k$, that is, every worker is able to produce one unit independently of how many workers are employed. Now the game is given by $v^{\prime \prime}(S)=|S|-1$ whenever $S \ni 0$ and otherwise $v^{\prime \prime}(S)=0 .{ }^{4}$

The employer and the workers can be considered as complementary inputs (capital and labor) of a production technology. In the first case, $v^{\prime}$, there is a shortage of capital, and the employer can press the wages down by playing out the workers against each other. In contrast, in the second case, $v^{\prime \prime}$, there is no shortage of capital, thus the employer and the workers are on equal terms in negotiations. Since the bargaining position of the employer is significantly stronger in the first situation, it seems plausible that he should obtain a higher payoff in $v^{\prime}$. However, any solution concept that assigns to the employer a higher payoff in $v^{\prime}$ than in $v^{\prime \prime}$ violates aggregate monotonicity, as $v^{\prime}(N)<v^{\prime \prime}(N)$ and $v^{\prime}(S)=v^{\prime \prime}(S)$ for all $S \varsubsetneqq N$. The aggregate monotonicity requirement in this context is less appealing.

\footnotetext{
4 These two games belong to the class of glove market games (Shapley, 1959; Apartsin and Holzman, 2003). We thank an anonymous referee for pointing out this fact.
} 


\section{Analysis}

We will now analyze how some solution concepts perform in the above example. Let $N=\{0,1,2,3\}$, let $0 \leq z \leq 1$, and suppose that $v_{z}$ is given by $v_{z}(0, i)=1, v_{z}(0, i, j)=2$ for all distinct $i, j \in\{1,2,3\}, v_{z}(N)=2+z$, $0 \leq z \leq 1$, and $v_{z}(S)=0$ for every other coalition $S$. Note that the functions $v^{\prime}$ and $v^{\prime \prime}$ in the above example coincide with $v_{z}$ for $z=0$ and $z=1$, respectively.

The core ${ }^{5}$ of $\left(N, v_{z}\right)$ contains payoff vectors that allocate to every worker $i=1,2,3$ a payoff between 0 and $z$ and to the employer the rest of the surplus, i.e.,

$$
\mathcal{C}\left(N, v_{z}\right)=\left\{\left(x_{0}, x_{1}, x_{2}, x_{3}\right)\left|\begin{array}{l}
x_{i} \in[0, z], i=1,2,3 \\
x_{0}=2+z-x_{1}-x_{2}-x_{3} .
\end{array}\right|\right\}
$$

Thus, with $z=0$ the core is a singleton, $\mathcal{C}\left(N, v_{0}\right)=\{(2,0,0,0)\}$, the unique payoff vector in the core assigns zero to each worker and the entire surplus, 2 , to the employer. For every $z>0, \mathcal{C}\left(N, v_{0}\right)$ is not a singleton and contains payoff vectors that assign positive payoffs to all workers. Notice that the core does not violate aggregate monotonicity on the set of games under consideration, since there is a selection in the core that is weakly increasing in $z \cdot{ }^{6}$ A simple example is the allocation $(2+z / 4, z / 4, z / 4, z / 4)$ that yields a strict improvement to all players as $z$ goes up.

The Shapley value is given by $S h_{0}\left(N, v_{z}\right)=\frac{5}{4}+\frac{z}{4}$ and $S h_{i}\left(N, v_{z}\right)=\frac{1}{4}+\frac{z}{4}$, $i=1,2,3$, and the per-capita nucleolus yields $\mathcal{P N}_{0}\left(N, v_{z}\right)=2+\frac{z}{4}$ and $\mathcal{P} \mathcal{N}_{i}\left(N, v_{z}\right)=\frac{z}{4}, i=1,2,3$. These two solution concepts assign increasing payoffs to all players as the worth of the grand coalition, $v_{z}(N)=2+z$, grows, thus obeying the aggregate monotonicity requirement. In contrast,

\footnotetext{
${ }^{5}$ We omit the definition of the core, as well as other solution concepts, referring to the classical literature, e.g., Maschler (1992).

${ }^{6}$ A set-valued solution is said to be aggregate monotonic if it possesses a single-valued selection that is aggregate monotonic.
} 
the nucleolus, which yields $\mathcal{N}_{0}\left(N, v_{z}\right)=2-\frac{z}{2}$ and $\mathcal{N}_{i}\left(N, v_{z}\right)=\frac{z}{2}, i=1,2,3$, is not aggregate monotonic, since the payoff to the employer decreases as $v_{z}(N)$ goes up. The kernel is not aggregate monotonic either: the described set of games $\left(N, v_{z}\right), 0 \leq z \leq 1$, belongs to the class of clan games where the nucleolus is a unique kernel element (Potters et al., 1989; Arin and Feltkamp, 1997).

Recall that a higher value of $z$ is less favorable for the employer: every worker knows that by refusing to work she can make the others lose $z$, so she can exert pressure on the employer in negotiations, and the pressure is higher when $z$ is larger. One can therefore expect from a solution to account for this attribute of the problem by assigning smaller payoffs to the employer for higher values of $z$, as the nucleolus and the kernel do. Thus, one who is convinced that the discussed attribute of the solution is essential should abolish monotonicity and avoid monotonic solutions, such as the Shapley value and the per-capita nucleolus.

\section{References}

Apartsin, Y. and R. Holzman (2003). The core and the bargaining set in glove-market games. International Journal of Game Theory 32, 189-204.

Arin, J. and V. Feltkamp (1997). The nucleolus and kernel of veto-rich transferable utility games. International Journal of Game Theory 26, 6173.

Aumann, R. J. (2000). Collected Papers. MIT Press.

Davis, M. and M. Maschler (1965). The kernel of a cooperative game. Naval Research Logistics Quarterly 12, 223-259. 
Grotte, J. H. (1970). Computation of and observations on the nucleolus and the central games. M.Sc. Thesis, Cornell University.

Hokari, T. (2000). The nucleolus is not aggregate-monotonic on the domain of convex games. International Journal of Game Theory 29, 133-137.

Maschler, M. (1992). The bargaining set, kernel, and nucleolus. In R. J. Aumann and S. Hart (Eds.), Handbook of Game Theory, Volume 1, pp. 591-667. North-Holland.

Maschler, M., B. Peleg, and L. Shapley (1971). The kernel and bargaining set for convex games. International Journal of Game Theory 1, 73-93.

Megiddo, N. (1974). On the nonmonotonicity of the bargaining set, the kernel and the nucleolus of a game. SIAM Journal on Applied Mathematics 2\%, $355-358$.

Moulin, H. and W. Thomson (1988). Can everyone benefit from growth? Two difficulties. Journal of Mathematical Economics 17, 339-345.

Potters, J., R. Poos, S. Tijs, and S. Muto (1989). Clan games. Games and Economic Behavior 1, 275-293.

Schmeidler, D. (1969). The nucleolus of a characteristic function game. SIAM Journal on Applied Mathematics 17, 1163-1170.

Shapley, L. S. (1959). The solutions of a symmetric market game. In A. W. Tucker and R. D. Luce (Eds.), Contributions to the Theory of Games, Vol. IV, Annals of Mathematics Studies 40, pp. 145-162. Princeton University Press.

Young, H. P. (1985). Monotonic solutions of cooperative games. International Journal of Game Theory 14, 65-72. 
Young, H. P., N. Okada, and T. Hashimoto (1982). Cost allocation in water resources development. Water Resources Research 18, 463-475. 